\title{
WILEY-VCH
}

(DOI: $10.1002 /(($ please add manuscript number $))$

Article type: Communication

\section{Flexible and Biofouling Independent Salinity Sensor}

Altynay Kaidarova, Marco Marengo, Giovanni Marinaro, Nathan R. Geraldi, Carlos M. Duarte, and Jürgen Kosel*

Prof. Jurgen Kosel, Marco Marengo, Giovanni Marinaro, Altynay Kaidarova

Computer, Electrical and Mathematical Sciences \& Engineering (CEMSE)

4700 King Abdullah University of Science and Technology(KAUST)

Kingdom of Saudi Arabia, Thuwal 23955

Email: jurgen.kosel@kaust.edu.sa

Dr.Nathan Geraldi, Prof. Carlos.M. Duarte

Red Sea Research Center (RSRC)

4700 King Abdullah University of Science and Technology (KAUST)

Kingdom of Saudi Arabia, Thuwal 23955

Keywords: Graphene, polyimide, flexible sensor, conductivity cell, salinity, biofouling

Salinity is one of the most relevant parameters in oceanography used to study properties of the oceans as well as the effects of climate change. Salinity measurements are challenging, due to the harsh environment that leads to corrosion and biofouling. In the context of animal monitors, salinity sensors should also be minimally intrusive and have a long lifetime. Here, a conductivity cell for salinity sensing is presented based on a single-step laser irradiation process on flexible polyimide substrate. The sensors are characterized by lightweight, flexibility, low power consumption and low fabrication costs. A two-electrode cell is used to measure the impedance, and thereby the conductivity, of the water in the $\mathrm{MHz}$ frequency range. It offers an accuracy of $\pm 0.5 \mathrm{psu}$, which is not affected by sensor deformation. Deployment of the sensors in the Red Sea revealed that the materials are corrosion resistant and can withstand the harsh environment. While biofouling is strongly affecting commonly employed low frequency conductivity measurements, in the $\mathrm{MHz}$ frequency range, it acts like a short-circuited capacitance. Hence, biofouling independent salinity sensing can be achieved using a two-electrode impedance measurement at a frequency of $1 \mathrm{MHz}$. 


\section{WILEY-VCH}

Human induced rapid environmental changes have negatively impacted marine environment, water quality and marine biodiversity. ${ }^{[1,2,3]}$ Shifts in oceanic physico-chemical properties induce drastic effects on marine communities worldwide. In this context, large and interdisciplinary efforts are currently directed toward the development of sensors capable of monitoring fundamental ocean properties. Salinity is a pivotal variable in the regulation of global circulation, impacting water column stability, ion concentration and speciation, air-sea exchange of gasses and functional properties of organisms. ${ }^{[4]}$ Traditionally, salinity is manually determined by collecting samples and forwarding them to land-based laboratories for chemical composition analysis, which is time- and labor- consuming ${ }^{[5]}$. The development of in situ biologging systems has allowed recording the conductivity, temperature, and depth (CTD) of the water from free-ranging animals as they swim through their environment ${ }^{[6],[7]}$. These devices are usually bulky, mechanically not flexible, fabricated using expensive multilayer screening or traditional micromachining /MEMS techniques and most importantly, not addressing the effect of biofouling ${ }^{[8],[9]}$ Indeed, the chemical composition of seawater, along with its biota, introduces two main challenges in salinity measurements, as seawater is (i) corrosive and (ii) rich in microorganisms that grow on solid surfaces and compromise sensor functioning ${ }^{[10]}$. Therefore, measurements conducted with existing solutions are not reliable on a long-term scale, since the attachment of microorganisms on the electrodes causes a drift of conductance towards lower values. Although work in literature highlights that the attachment of organisms to the electrodes' surface could affect the measurement, none has addressed this issue. In addition, an increased interest in sensors on flexible substrates that can be attached to the curved surfaces of marine animals or follow their deformation, and which are small in size, lightweight and minimally intrusive cannot be met with the existing CTD devices. 


\section{WILEY-VCH}

The "rise of graphene" in 2006 prompted the development of numerous graphene-based sensors, because of its outstanding mechanical, electrical and thermal properties. ${ }^{[11,12]}$ Recently, Lin et el. studied $\mathrm{CO}_{2}$ laser irradiation of polyimide (PI), and thoroughly characterized the carbonization process of $\mathrm{PI}^{[13]}$. They proved that that laser technology can be used to fabricate patterned conductive porous carbon networks with high content of graphene sheets; thereby, they named the material laser-induced graphene (LIG). The cheap single-step fabrication of LIG on a flexible substrate has already been exploited for strain and electrochemical sensors ${ }^{[14-16]}$. The corrosion resistance of graphene has also been shown ${ }^{[19]}$ and it was used as electrode in saline solutions ${ }^{[17-19]}$. In addition, LIG has been associated with voltage dependent, antimicrobial action, ${ }^{[20]}$ making it a suitable candidate for marine applications. Consequently, LIG has recently been utilized for capacitive salinity sensing, realized by a two-step fabrication process, where graphene was first thermally-induced on a PI film and then transferred to a thicker substrate by applying manual pressure to the electrodes. ${ }^{[21]}$. Here, we report a flexible conductivity cell made by a single LIG fabrication step. Moreover, we propose a two-electrode, high frequency impedance measurement for biofouling independent salinity sensing systems.

Ions present in a saline solution are responsible for the electrical conduction; therefore, the conductivity is directly correlated to the concentration of salt. Typically, a current, I, is driven through the solution and the potential drop, V, is measured to obtain the conductance by

$$
G=\frac{I}{V}
$$

Such measurement is carried out by immersing two-electrodes in the saline solution, thereby forming a conductivity cell. Randles equivalent circuit (Figure 1a) is commonly used to design an electrical model of the conductivity cell, taking into consideration the main parasitic components, i.e. (i) the formation of an electrical double layer on the electrode-solution interface, (ii) charge transfer phenomena caused by red-ox processes, and (iii) diffusion of 


\section{WILEY-VCH}

ions close to the interface. The measured conductance is then multiplied by the cell constant $\mathrm{K}$, a parameter related to the geometry of the cell, thereby obtaining the conductivity $(k)$, which is the intrinsic property of the solution and does not depend on cell geometry. The cell constant in a two pole probe is given by

$$
K=\frac{\text { distance between the electrodes }}{\text { surface area in contact with the solution }},
$$

whereas for planar electrodes and complex geometries the cell constant must be calculated through software simulation, as reported by Hyldgård, et.al ${ }^{[8]}$. Since the conductance measured depends on the surface area, it is evident that biofouling can affect such measurement method. Indeed, after examining the performance of commonly used fourelectrode conductivity cells (see Figure $\mathbf{S 1}$ in supplementary materials) and deployment of them in the Red sea for 3 weeks, we found that bioufouling has induced a shift in the conductance towards lower values (see Figure S2 in supplementary materials). This is because the area exposed to seawater decreased by biofouling, leading to an increase of the cell constant and decrease in conductance. Electrochemical impedance spectroscopy (EIS) is largely used for biosensors, such that a change in the conductance of the growth medium provides information about microbial metabolism. Sergi Brosel-Oliu et al. studied how a solution with microorganisms can affect EIS curve and how Randles equivalent circuit modifies accordingly. ${ }^{[22]}$ They reported that the attachment of bacteria on electrodes' surfaces increases the charge-transfer resistance and adds a capacitor in series or in parallel to the double-layer capacitor, depending on the amounts of bacteria attached. However, media conductance effects are predominant at high frequency. ${ }^{[23,24]}$ We exploit this concept with a two-electrode cell to obtain biofouling independent salinity measurements for extended periods of time in harsh seawater environment. Specifically, we are measuring the conductance of the solution, while avoiding the influence of foulants' recruitment on the 


\section{WILEY-VCH}

surface of the electrodes by a high frequency impedance measurement, which is therefore expected to solve long-term reliability issues.

Two-electrodes were directly patterned on PI film using a $\mathrm{CO}_{2}$ infrared laser in ambient conditions (Figure 2a). During laser irradiation, the PI film was locally heated, leading to C$\mathrm{O}, \mathrm{N}-\mathrm{C}$, and $\mathrm{C}=\mathrm{O}$ bonds breaking with release of gaseous byproducts and rearrangement of the carbon atoms, forming the porous structure, shown in Figure $\mathbf{2 b , c , d}$. The Raman spectrum of LIG (Figure 2e) shows 3 main peaks D, G and 2D at 1360, 1580 and $2720 \mathrm{~cm}^{-1}$, respectively, that are in accordance with previous studies and entail the presence of graphene sheets in the porous structure. ${ }^{[16]}$ The $\mathrm{G}$ peak is related to the vibration of $\mathrm{sp}^{2}$ carbon atoms in the hexagonal plane, and its intensity is lower in mono-layer graphene. ${ }^{[25]}$ The D peak is activated by defects and correspond to intravalley double resonance processes. ${ }^{[26]}$ The $2 \mathrm{D}$ peak is the main one in monolayer graphene and it decreases and broadens as the number of layers increases. ${ }^{[27]}$

For the sake of coherence with previous works, we use the practical salinity unit (psu) to express salinity of seawater. The chosen six solutions (10, 18, 26, 34, 42 and 50 psu) allowed characterizing the sensor in a range of salinities that are typical for most of the water masses of the planet, both sea and oceans.

Figure 3a reveals that the impedance of the two-electrode conductivity cell decreases as a function of frequency. In the lower frequency range (Figure S3a), the impedance slightly varies with the salinity and shows a poor correlation. On the other hand, at high frequencies the impedance strongly depends on variations of the salt concentration (Figure S3b), and the phase reaches approximately $0^{\circ}$ at $10 \mathrm{MHz}$ (Figure 3b). This confirms that the impedance at high frequencies is related to the medium's conductance, whereas capacitive, diffusive, and charge transfer effects are negligible. The hypothesis is supported by simulating Randles equivalent circuit using LTSpice, wherein we substituted the Warburg impedance with a capacitor $\mathrm{C}_{z}$ (Figure 3c). Using the component values of Table S1, a good agreement with 


\section{WILEY-VCH}

the experimental curve is obtained, as shown in Figure 3 d, e. Since at high frequencies the impedance is given by the sum of the resistances of the electrodes and of the saline solution, parasitic effects, including biofouling, will be minimized in the response of the sensor. The impedance, at $1 \mathrm{MHz}$, as a function of salinity is shown in the Figure 4, where the frequency was chosen arbitrarily, since the measurement is frequency independent within the range of $1 \mathrm{MHz}$ to $10 \mathrm{MHz}$. The results confirm that the impedance at high frequency is inversely proportional to the salinity.

The response of the flexible two-electrode conductivity cell under bending was evaluated by gluing a sensor onto the outer wall of a round plastic tube with a radius of $1 \mathrm{~cm}$ (Figure 4). The measured values are consistent with the planar electrode, indicating that the sensor's performance is maintained upon bending and operation of the conductivity cell under various mechanical conditions is feasible. Compared to a planar conductivity cell, the impedance values of a bent cell have decreased by $\sim 1.05 \%$, due to geometrical factors. The porous graphene structure was stretched causing a larger electrodes area exposure to the saline solution. The reduced cell constant led to a slight increase in the measured conductance and decrease in the impedance.

The temperature dependence of nine sensors (Figure S5a) was examined according to the procedure described in the experimental section. As shown in Figure S5b, the average values of the conductance increase linearly with temperature with a coefficient of determination $\left(\mathrm{R}^{2}\right)$ of 0.9269 . Temperature affects the salinity measurements, as the mobility of the ions increases with temperature. ${ }^{[28]}$ A common way to evaluate the accuracy of the sensor is by fitting the experimental result to the theoretical curve based on the UNESCO technical papers in marine science ${ }^{[29]}$. The fitting requires to calculate the conductivity, which is obtained by multiplying the measured conductance by the cell constant. A cell constant of $\mathrm{K}=5.2 \mathrm{~cm}^{-1}$ provides a good fit, and was used to obtain the result shown in Figure 5. The measurement results follow the theoretical curve with an accuracy of $0.5 \mathrm{psu}$. 


\section{WILEY-VCH}

In order to conduct a biofouling study and investigate biofoulants' recruitment on LIG and their effect on the performance of the sensor, the sensors were deployed in a $0.125 \mathrm{~m}^{3}$ aquarium filled with sea water from the Red Sea for up to 3 weeks. The samples were attached to the side walls of the aquarium facing living seawater marine animals (giant clams Tridacna gigas), such that microorganisms were allowed to settle on top of the sensors. This provided an extreme test of corrosion and biofouling, because of the hypersaline nature of Red sea water (salinity > 39) and the rapid recruitment and growth of biofouling organisms. ${ }^{[30]}$ This period of time was needed for the fouling process to be substantial, since biofouling is constituted by three main phases: (i) conditioning film, (ii) microfouling and (iii) macrofouling. ${ }^{[31]}$

Figure 6 shows the measurement results of three two-electrode LIG salinity sensors before deployment and after 1-3 weeks in the seawater (Individual device performances is reported in Figure S6) Using ImageJ software ${ }^{[32]}$, the area of the graphene surface covered by biofoulants was found to be $\sim 13 \%, \sim 24 \%$ and $\sim 37 \%$ after 1,2 and 3 weeks respectively. The amount of biofouling after one, two and three weeks was also observed using fluorescent stereomicroscope as shown Figure S7. Nevertheless, the impedance values measured at 1 $\mathrm{MHz}$ before and after exposure to seawater are very similar and clearly enable discerning different salinities, without being affected by the biofouling. This confirms the assumption that the biofouling layer attached to the LIG electrodes acts as an additional capacitor, which has a negligible contribution to the impedance at high frequencies. The total capacitor $\left(\mathrm{C}_{\mathrm{t}}\right)$, shown in Figure 3c , is associated with a parallel configuration of the double layer $\left(\mathrm{C}_{\mathrm{dl}}\right)$ and the biofouling capacitor $\left(\mathrm{C}_{\mathrm{bf}}\right)$. Some differences between the values before and after deployment are attributed to the connection with the tweezers used to carry out the measurement with the impedance analyzer, which affects the calibration and contact resistance. However, the response does not show an overall shift of the values, thereby 


\section{WILEY-VCH}

confirming that high frequency impedance spectroscopy allows achieving long-lasting and reliable salinity sensing systems.

In summary, the sensor shown in this manuscript, consisting of laser-induced graphene electrodes on polyimide, is flexible, cheap, lightweight and operates under various bending conditions. Moreover, the single-step fabrication method by laser writing is a key feature, which enables prototyping and mass fabrication. Benefiting from the versatility of the laser writing technology, the size of the sensor can be easily tailored and further reduced to allow integration of the sensor on CTD devices for small-sized marine animals. For long-term measurements, a biofouling study revealed that LIG does not prevent biofoulants' attachment to the surface in a real seawater environment, which strongly affects the measurement results in commonly used low frequency setups. In order to overcome this issue, we investigated high frequency impedance measurements with a two-electrodes conductivity cell. We found that this measurement is not affected by the biofouling and reliably measures salinity even after 3 weeks of deployment in the waters of the Red Sea.

\section{Experimental Section:}

Materials: $125 \mu \mathrm{m}$ thick PI films (Kapton \# IM301449, DuPont, Delaware, USA) and $\mathrm{CO}_{2}$ infrared laser (Universal Laser Systems® PLS6.75 $10.6 \mu \mathrm{m}$, laser peak power $75 \mathrm{~W}$ ) were utilized to fabricate electrodes. The main parameters of the laser beam were $2 \mathrm{~W}$ power, 1.6 $\mathrm{cm} / \mathrm{s}$ speed, 1000 pulses per inch and $3 \mathrm{~mm}$ working distance. The parameters were optimized for good adhesion of the carbon structure to the substrate.

Characterization: Confocal Raman measurements were carried out on Horiba Aramis using a laser of $473 \mathrm{~nm}$ in room temperature. Cross sections of the sensors were observed by SEM using Quanta 600FEG Systems.

Sample preparation: After the fabrication of the electrodes, the sensor was attached to a $2 \mathrm{~mm}$ thick polymethylmethacrylate (PMMA) sheet with instant adhesive glue (406, Henkel Loctite, UK) to provide a stable support. Then single-sided copper foil tape (1/4 inch x 21.8 yards, 


\section{WILEY-VCH}

Kraftex Inc., UK) was manually applied on LIG contact pads and electrically conductive silver epoxy (EMS \#12642-14, Electron Microscopy Sciences, USA) was used to stabilize the contacts. Kapton tape and instant glue were used to define the area of the electrodes that were immersed in solution and to protect the pads from the contact with saline water (Figure S4). Saline solutions: 1 psu corresponds to 1 gram of salt per liter of solution. Solutions are prepared by mixing $\mathrm{NaCl}$ and $100 \mathrm{ml}$ of deionized water $(18.2 \mathrm{M} \Omega \cdot \mathrm{cm}$, Milli-Q Direct 8 , Merck Millipore and Billerica, MA).

Conductivity measurement procedure: The sensor was first washed in deionized water, then soaked in one solution for 3 minutes (starting from the lowest concentration), characterized and subsequently washed again in deionized water and dried with nitrogen flow to avoid contaminating the next solution. The spectrum (EIS) was obtained using an impedance analyzer (Agilent 4294A) in combination with a 16334A Test Fixture (Tweezer Contacts) and the sensors were characterized in the range of $100 \mathrm{~Hz}$ to $10 \mathrm{MHz}$. The impedance and phase were measured as functions of the frequency with an AC current of $1 \mathrm{~mA}$ (rms value). A low driving current was chosen to minimize electrolysis of seawater, which may induce air bubbles on the electrodes' surfaces, thereby altering the value of the cell constant.

Measurement of temperature dependence: Nine sensors were prepared using the same laser parameters to investigate the temperature dependence of the measured conductance. The sensors were immersed in a 34 psu solution over night at $4^{\circ} \mathrm{C}$. Then, the temperature was slowly increased until it reached room temperature and the impedance was measured every $2^{\circ}$. The temperature was monitored using a Digital Temperature Controller (HY-8000S, HANYOUNG CO.,LTD).

\section{Supporting Information}




\section{WILEY-VCH}

Supporting Information is available from the Wiley Online Library or from the author.

\section{Acknowledgements}

This research is a contribution to the CAASE project funded by King Abdullah University of Science and Technology (KAUST) under the KAUST Sensor Initiative. The author would like to thank Ulrich Buttner from the KAUST Microfluidics Core Laboratory for his technical support.

Received: ((will be filled in by the editorial staff))

Revised: ((will be filled in by the editorial staff)) Published online: ((will be filled in by the editorial staff))

\section{References}

1. T.P. Hughes, A.H. Baird, D.R Bellwood, M. Card, S.R. Connolly, C. Folke, R. Grosberg, O . Hoegh-Guldberg, J.B. Jackson, J. Kleypas, and J.M. Lough, Climate change, human impacts, and the resilience of coral reefs. Science 2003, 301(5635), pp.929-933.

2. R.A. Feely, C.L. Sabin, K. Lee, W. Berelson, J.Kleypas , V.J Fabry, F.J. Millero. Impact of anthropogenic $\mathrm{CO} 2$ on the $\mathrm{CaCO} 3$ system in the oceans. Science 2004, 16;305(5682), pp.362-6.

3. S.C. Doney, The growing human footprint on coastal and open-ocean biogeochemistry. Science 2010, 328, pp.1512-1516 .

4. H.A. Broadbent, S.Z. Ivanov, D.P. Fries, A miniature, low cost CTD system for coastal salinity measurements. Meas. Sci. Technol 2007,. 18, pp.3295-3302

5. A. Hounslow, Water quality data: analysis and interpretation, CRC press 2018.

6. S.K. Hooker, I.L.Boyd, Salinity sensors on seals: use of marine predators to carry CTD data loggers. Deep Sea Res. Part I Oceanogr. Res. Pap 2003, 50, pp. 927-939

7. G. Mills, G.A Fones, Review of in situ methods and sensors for monitoring the 


\section{WILEY-VCH}

marine environment. Sens. Rev 2012, 32, 17-28.

8. A. Hyldgård, D. Mortensen, K. Birkelund, O.Hansen, E.V Thomsen, Autonomous multi-sensor micro-system for measurement of ocean water salinity. Sensors Actuators, A Phys 2008, 147, 474-484.

9. L. Delauney, C. Compere, Biofouling protection for marine environmental sensors. Ocean Science 2010, 6(2), pp. 503-511.

10. A.G. Nurioglu, A.C. Esteves, Non-toxic, non-biocide-release antifouling coatings based on molecular structure design for marine applications. Journal of Materials Chemistry B 2015, 3(32), pp.6547-6570.

12. K.S. Novoselov, V.I Fal, L. Colombo, P.R. Gellert, M.G Schwab, K. Kim, A roadmap for graphene. Nature 2012, 490(7419), pp.192.

13. J.Lin, Z.Peng, Y. Liu, F.Ruiz-Zepeda, R.Ye, E.L. Samuel, M.J. Yacaman, B.I. Yakobson, J.M Tour, Laser-induced porous graphene films from commercial polymers, Nature communications 2014, 5. pp. 5714.

14. H.Tian, Y.Shu, Y.L.Cui, W.T. Mi, Y. Yang, D.Xie, T.L.Ren,Scalable fabrication of high-performance and flexible graphene strain sensors. Nanoscale 2014, 6(2), pp.699705.

15. S. Luo, P.T. Hoang, T. Liu, Direct laser writing for creating porous graphitic structures and their use for flexible and highly sensitive sensor and sensor arrays. Carbon N. $Y$ 2016, 96, pp. 522-531.

16. P.Nayak, N. Kurra, C. Xia, H.N. Alshareef, Highly Efficient Laser Scribed Graphene Electrodes for On-Chip Electrochemical Sensing Applications. Adv. Electron. Mater 2016. 2 (10), pp. 1600185.

17. D.Prasai, J.C. Tuberquia, R.R. Harl, Jennings, K.I. Bolotin, Graphene: CorrosionInhibiting Coating. ACS Nano 2012, 6, 1102-1108.

18. M.A.Khan, I.R Hristovski, G.Marinaro, J.Kosel, Magnetic composite Hydrodynamic 


\section{WILEY-VCH}

Pump with Laser Induced Graphene Electrodes. IEEE Trans. Magn. 2017, 53(11), pp.1-4

19. M.A. Khan, I.R. Hristovski, G. Marinaro, H.Mohammed, J.Kosel, Laser printed graphene on polyimide electrodes for magnetohydrodynamic pumping of saline fluids. In Solid-State Sensors, Actuators and Microsystems (TRANSDUCERS) 2017, 19th International Conference on, pp. 1387-1390.

20. S.P. Singh, Y.Li, A. Be'er, Y. Oren, J.M. Tour, C.J. Arnusch, Laser-induced graphene layers and electrodes prevents microbial fouling and exerts antimicrobial action. ACS applied materials \& interfaces 2017,9(21), pp. 18238-47.

21. A.Nag, S.C. Mukhopadhyay, J.Kosel, Sensing System for Salinity Testing Using Laser-induced Graphene Sensors. Sensors Actuators A Phys .2017, 264, pp.107-116

232. S.Brosel-Oliu, N. Uria, N. Abramova, A.Bratov, in Biosensors-Micro and Nanoscale Applications 2015, InTech.

23. C.J.Felice, M. E.Valentinuzzi, M.I.Vercellone, R.E. Madrid, Impedance bacteriometry: medium and interface contributions during bacterial growth. IEEE Trans. Biomed. Eng.1992, 39, pp.1310-1313.

24. L.L.Hause, R.A. Komorowski, F. Gayon, Electrode and electrolyte impedance in the detection of bacterial growth. IEEE Trans. Biomed. Eng 1981. pp. 403-410

25. T. Kuila, S.Bose, A.K. Mishra, P. Khanra, N.H. Kim, J.H.Lee, Chemical functionalization of graphene and its applications. Progress in Materials Science 2012, 57(7), pp.1061-105.

26. L.G.Cançado, A. Jorio, E.M.Ferreira, F.Stavale,C.A. Achete,R.B. Capaz, M.V. Moutinho, A.Lombardo, T.S.Kulmala,A.C. Ferrari, Quantifying defects in graphene via Raman spectroscopy at different excitation energies. Nano letters 2011, 11.8, pp. 31903196.

27. A.C.Ferrari, J.C.Meyer, V. Scardaci, C.Casiraghi, M. Lazzeri , F. Mauri, S. Piscanec, 


\section{WILEY-VCH}

D.Jiang, K.S. Novoselov, S. Roth, A.K. Geim. Raman spectrum of graphene and graphene layers, Physical review letters 2006, 97(18), pp. 187401.

28. R.C Hunt How to increase the accuracy of solution conductivity measurements. Sensor Development Inc. 1995,(1).

29. R. C. Millard, and N. P. Fofonoff, Algorithms for computation of fundamental properties of seawater. Unesco 1983.

30. R.E. Spieler, D.S. Gilliam, R.L.Sherman, Artificial substrate and coral reef restoration: what do we need to know to know what we need. Bull. Mar. Sci 2001. 69, pp. 1013-1030.

31. A.G.Nurioglu, A. Non-toxic, non-biocide-release antifouling coatings based on molecular structure design for marine applications. J. Mater. Chem. B. 2015. 3(32), pp. 6547

32. M.D. Abràmoff , P.J. Magalhães PJ, S.J. Ram SJ. Image processing with ImageJ. Biophotonics international 2004, 11(7), pp.36-42. 


\section{WILEY-VCH}
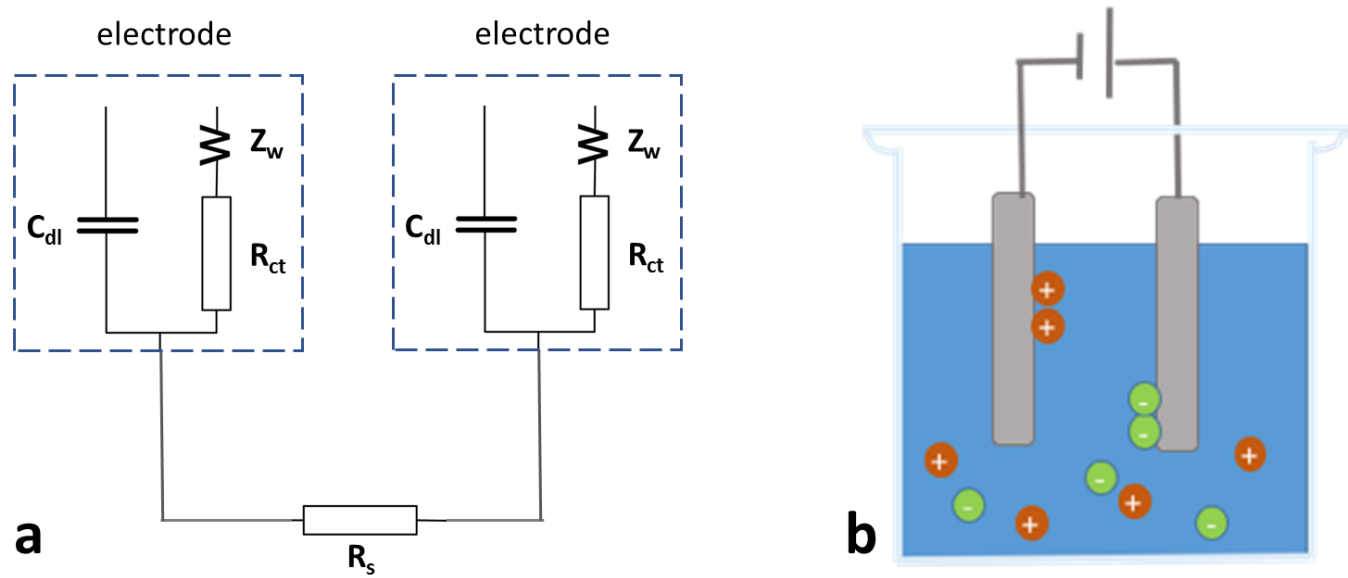

Figure 1. a) Randles equivalent circuit used to model parasitic phenomena in a twoelectrodes cell. The electrode/solution interface consists of a parallel connection of the double-layer capacitor $\left(\mathrm{C}_{\mathrm{dl}}\right)$ and the charge-transfer resistor $\left(\mathrm{R}_{\mathrm{ct}}\right)$ in series with the Warburg impedance $\left(Z_{w}\right)$. The two electrodes are connected in series with the solution's resistor $\left.\left(R_{s}\right) b\right)$ Two- electrode conductivity cell system constituted by electrodes soaked in a saline solution.
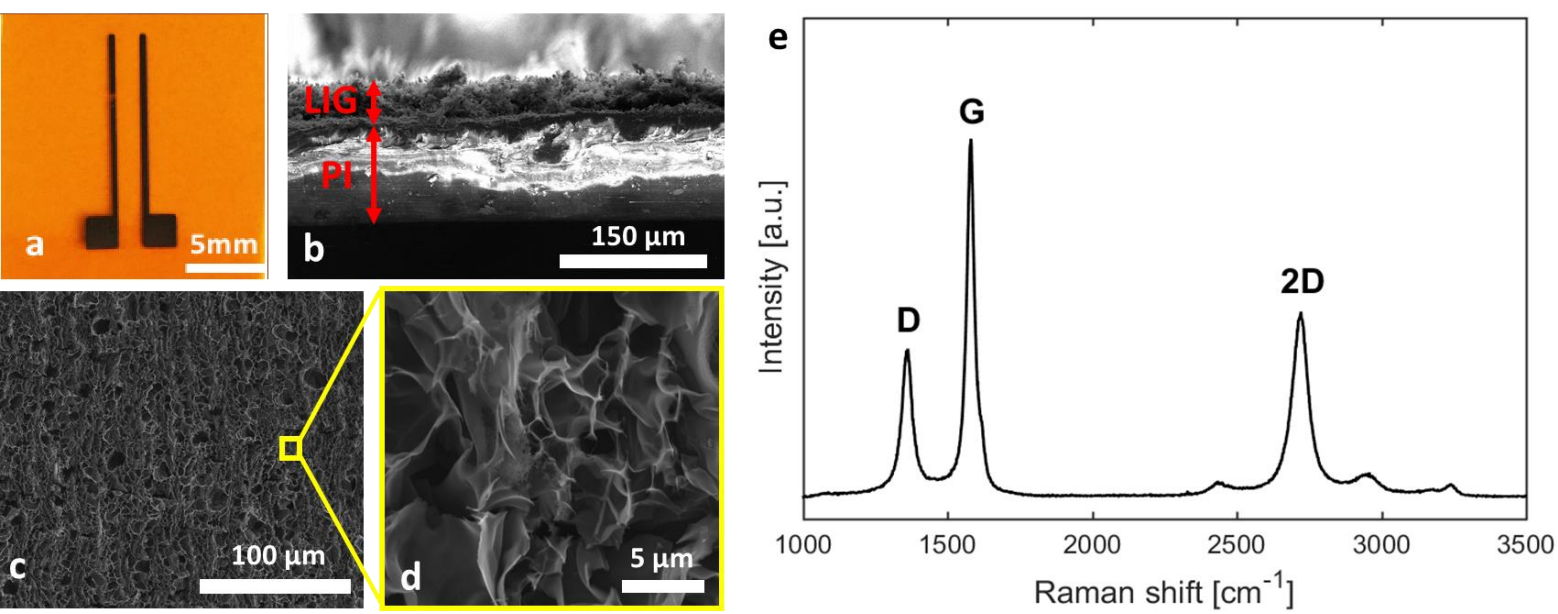

Figure 2. a) LIG electrodes (black) patterned on flexible polyimide sheet (orange). b) SEM image of a cross section showing the porous carbon network laying on top of polyimide. c) Low magnification SEM image shows the porous morphology of LIG. d) High magnification SEM image shows the graphene flakes randomly arranged and interconnected. e) Raman spectrum of LIG acquired with a laser wavelength of $473 \mathrm{~nm}$. 


\section{WILEY-VCH}
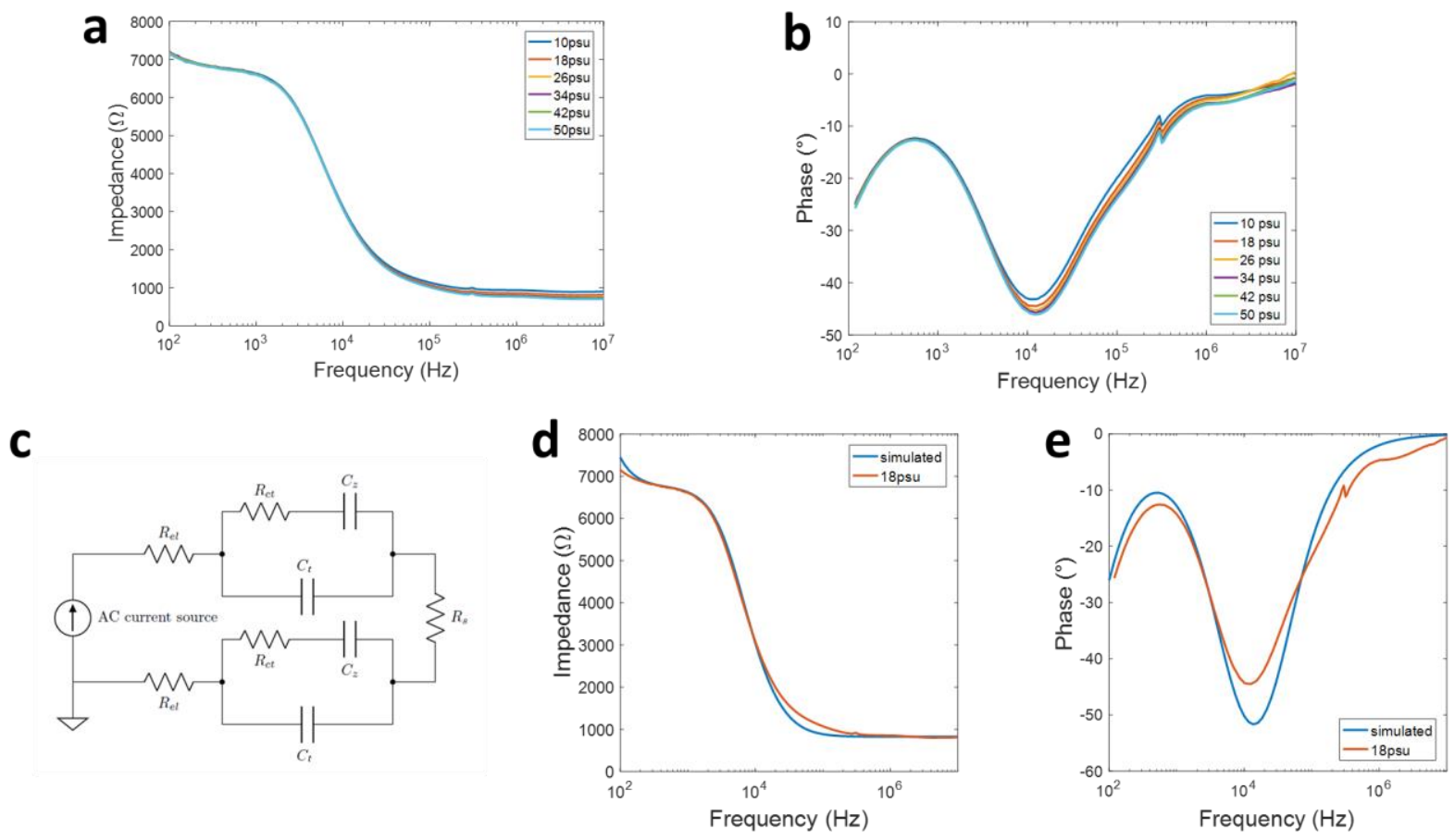

Figure 3. a) Impedance and b) phase measured as a function of frequency and salinity with a two-electrodes conductivity cell. c) Scheme of the equivalent circuit used for the simulation with LTSpice, where electrode resistors $\left(\mathrm{R}_{\mathrm{el}}\right)$ are connected in series with the solution resistor $\left(R_{S}\right)$ and a parallel circuit of total capacitance $\left(C_{t}\right)$, charge-transfer resistor $\left(R_{c t}\right)$ and Warburg element $\left(C_{z}\right) . C_{t}$ is the equivalent capacitance associated with the double-layer $\left(C_{d l}\right)$ and biofouling $\left(\mathrm{C}_{\mathrm{bf}}\right)$ layer. d) Impedance over frequency obtained by simulation and experimentally e) Phase as a function of frequency obtained by simulation and experiment.

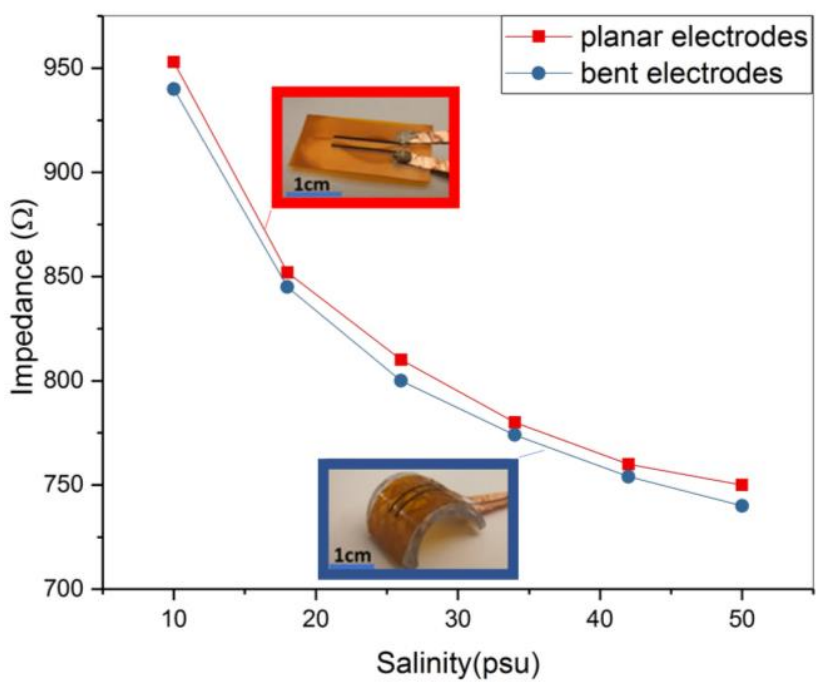

Figure 4. Sensor impedance as a function of salinity for the planar and bent $(1 \mathrm{~cm}$ radius of curvature) configurations. The insets show photographs of the sensors. 


\section{WILEY-VCH}

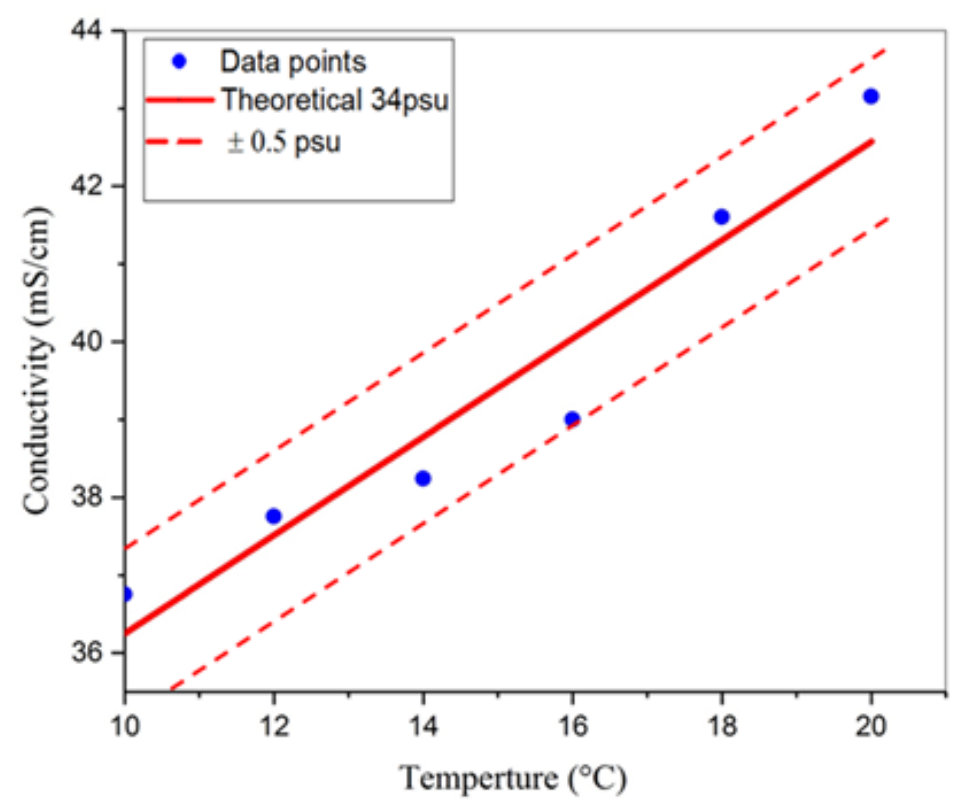

Figure 5. Conductivity as a function of temperature of the LIG conductivity cell compared to the theoretical curve based on UNESCO papers.

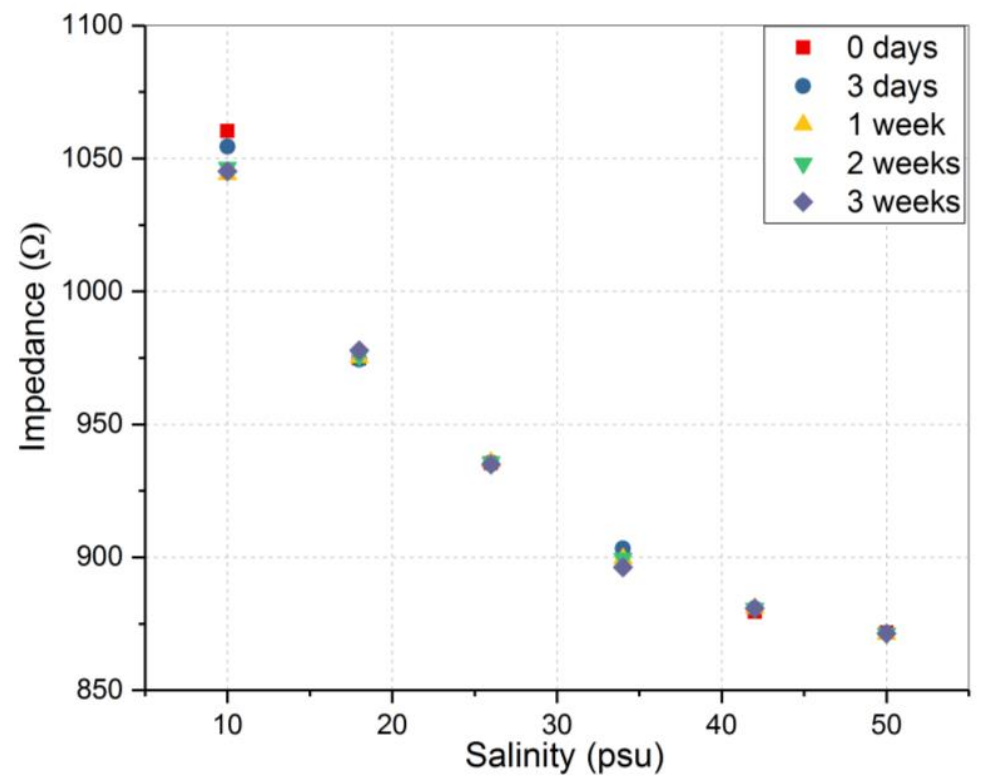

Figure 6 Average response of two-electrode conductivity cells at high frequency $(1 \mathrm{MHz})$ before deployment and after different periods of time in seawater. 


\section{WILEY-VCH}

Copyright WILEY-VCH Verlag GmbH \& Co. KGaA, 69469 Weinheim, Germany, 2016.

\section{Supporting Information}

\section{Flexible and Biofouling Independent Salinity Sensor}

Altynay Kaidarova, Marco Marengo, Giovanni Marinaro, Nathan Geraldi, Carlos M. Duarte, and Jürgen Kosel*

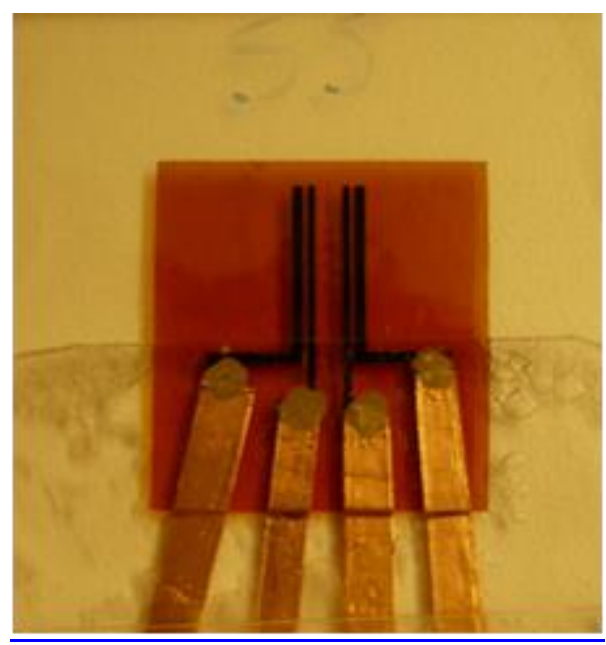

Figure S1: a) Assembled four-electrode conductivity cell prior to deployment in the Red Sea for a biofouling study.
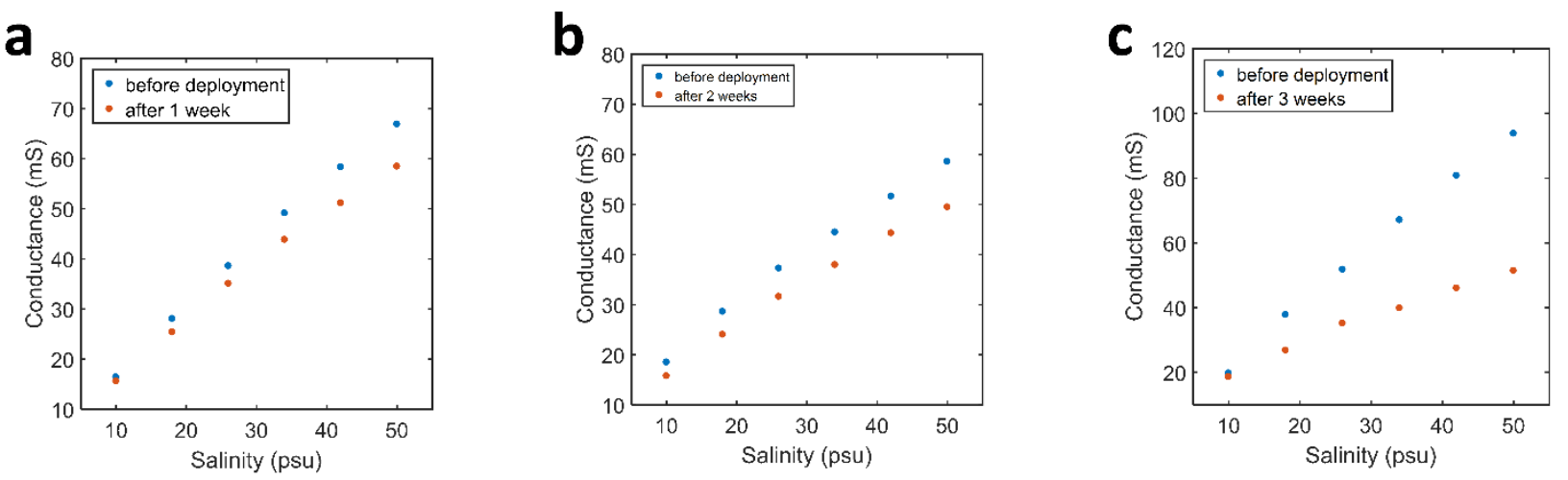

Figure S2: Conductance measurement of four electrode cell before and after deployment in seawater to evaluate the effect of biofouling. 
WILEY-VCH
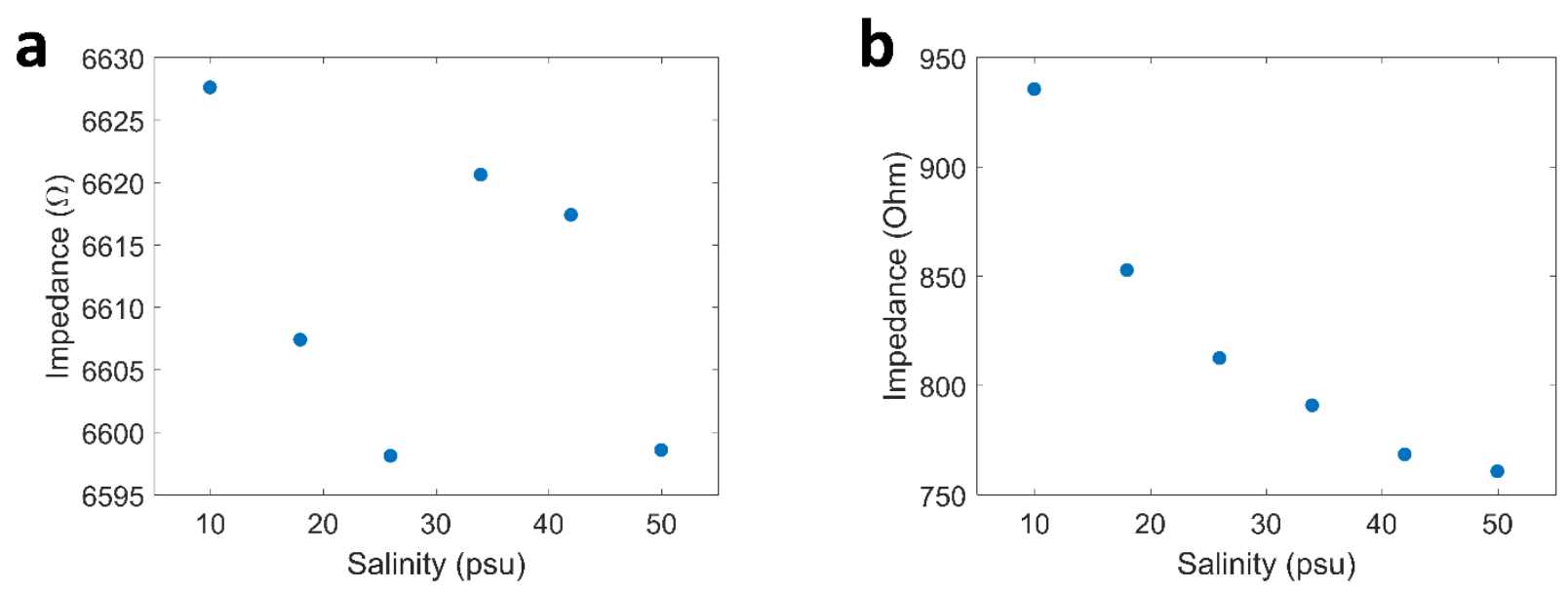

Figure S3: Impedance extracted from the EIS spectrum at $1 \mathrm{kHz}$ (a) and $1 \mathrm{MHz}$ (b).

Table S1: Simulation with LTSpice of Randles equivalent circuit gives the values of the components which allow to fit the experimental curve.

\begin{tabular}{|c|c|}
\hline $\mathrm{R}_{\mathrm{el}}$ & $350 \Omega$ \\
\hline $\mathrm{R}_{\mathrm{ct}}$ & $3000 \Omega$ \\
\hline $\mathrm{C}_{\mathrm{z}}$ & $1 \mathrm{uF}$ \\
\hline $\mathrm{C}_{\mathrm{dl}}$ & $11 \mathrm{nF}$ \\
\hline $\mathrm{R}_{\mathrm{s}}$ & $150 \Omega$ \\
\hline Current Source & $1 \mathrm{~mA}(100 \mathrm{~Hz}-10 \mathrm{MHz})$ \\
\hline
\end{tabular}

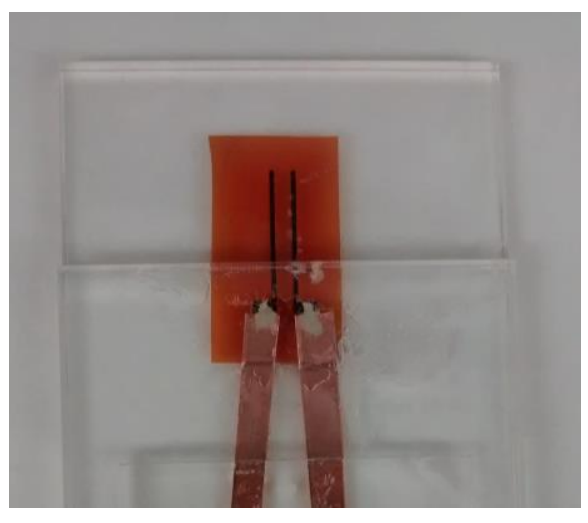

Figure S4: Fully assembled LIG conductivity sensor 


\section{WILEY-VCH}

\section{a)}

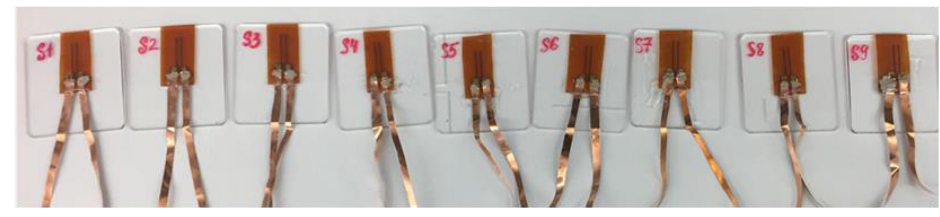

b)

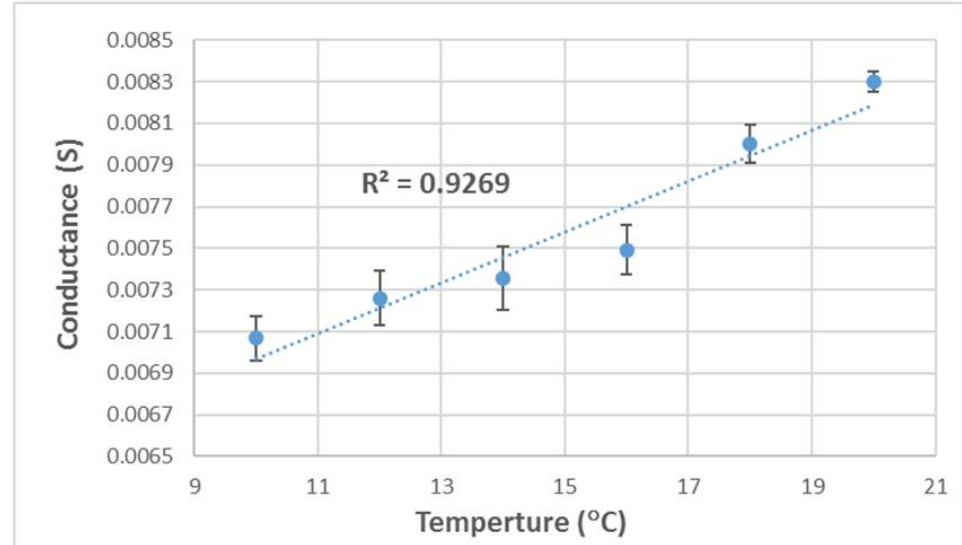

Figure S5 a) Sensors fabricated with the same laser parameters and using the same fabrication procedure $b$ ) Average conductance as a function of temperature. The error bars indicate the standard deviation. The coefficient of determination $\left(\mathrm{R}^{2}\right)$ is 0.9269 .
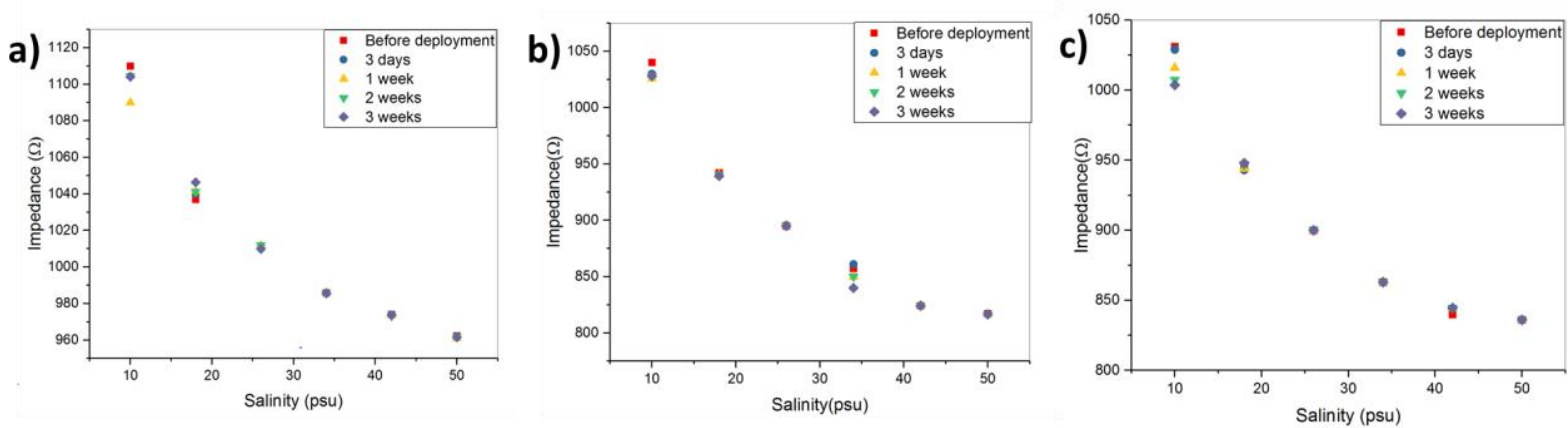

Figure S6 a-c) Response of three two-electrode conductivity cells at high frequency before deployment and after different periods of time in seawater.
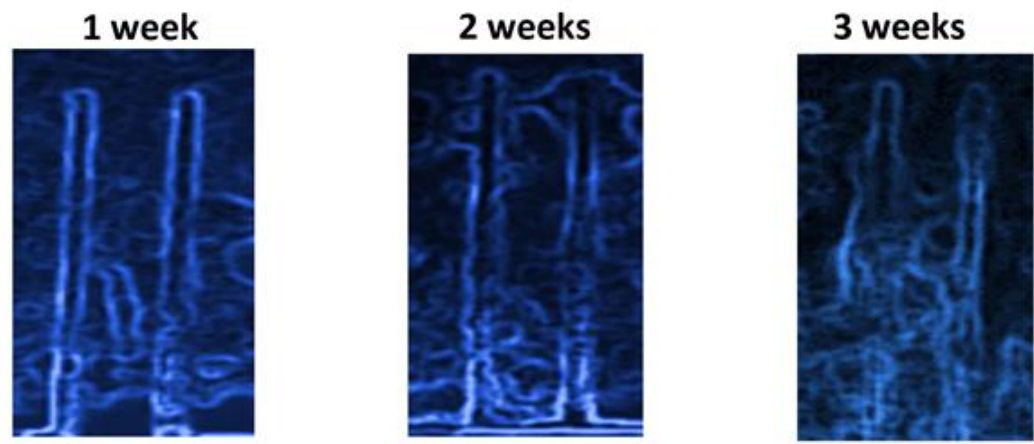

Figure S7. The accumulation of biofouling after one, two and three weeks observed using a fluorescent stereomicroscope (Leica MZ16 F, Meyer Inc.,) 